\title{
New data acquisition control software developments for the simultaneous control of ten intelligent overview video cameras at $\mathrm{W} 7-\mathrm{X}$
}

Tamás Szabolicsa ${ }^{\mathrm{a}}$, Gábor Cseh ${ }^{\mathrm{a}}$, Gábor Kocsis ${ }^{\mathrm{a}}$, Tamás Szepesi ${ }^{\mathrm{a}}$, Sándor Zoletnik ${ }^{\mathrm{a}}$ Christoph Biedermann ${ }^{\mathrm{b}}$, Ralf König ${ }^{\mathrm{b}}$, Aleix Puig Sitjes ${ }^{\mathrm{b}}$, Torsten Bluhm ${ }^{\mathrm{b}}$ and the W7-X team ${ }^{\mathrm{b}}$

\author{
${ }_{4}^{a}$ Wigner RCP, RMI, Konkoly-Thege 29-33, H-1121 Budapest, Hungary \\ ${ }^{b}$ Max-Planck-Institut für Plasmaphysik, Wendelsteinstraße 1, D-17491, Greifswald, German
}

Formatiert: Englisch (Vereinigte Staaten)

In the past two campaigns of Wendelstein 7-X (W7-X) stellarator the overview video diagnostics played a key role in the daily experiments. The current software implementations went through numerous improvements and changes according to the continuously changing requirements. However, while the control software could handle all the needs, the changes reached a point where the redesign and re-implementation became necessary to ensure high reliability. In the next campaion of W7-X real-time data streaming will be implemented to W7-X's Archive, which can be practically a limitless real-time data backup. Other improvements involve high-level interaction with the trigger-time-event (TTE) network, intense graphical user interface (GUI) improvements such as the grouping of cameras, visual display of measurement settings (e.g. exposure and readout timing). This new software package is arranged into two standalone pieces, similarly as in the previous version. One of the main upgrades is that the software will be able to send so-called event descriptions over the network during the measurement to a central event display, where events from several diagnostics will be visualized. This feature can provide essential information for machine operators, as they can visually monitor dangerous events inside the machine in real-time. This paper will present the detailed experiences, new requirements and detailed design, implementation and testing of this new software package.

Keywords: Video Diagnostics, Event Detection Intelligent Camera, EDICAM, Wendelstein 7-X, W7-X, Data acquisition and control software, Software development

\section{Introduction}

Wendelstein 7-X [1] is the world's newest, largest and the most complex stellarator type fusion device which needs high performance diagnostics. One of them is the video diagnostics, operating since the first experiments started back in 2015. The purpose of the video diagnostics system is to monitor the plasma in millisecond or shorter time scales; consequently, it can provide a huge amount of data in a very short time. The maximum data rate of a single camera is $950 \mathrm{MB} / \mathrm{s}$.. The Event Detection Intelligent Camera (EDICAM) was developed to be able to handle and process this data flow in real-time, and to make decisions without human interaction [2]. The observation system has 10 equatorial ports to monitor the whole interior of the machine and in Op1.2 eight of these were equipped with EDICAMs.

In this paper a summary of the old system, the new requirements, software solutions of the new video diagnostics control software system, design details, test results and first campaign experiences are described. The old control software details, camera concept and specification were published in a previous paper [3]

\section{The old system}

The previous version of the video diagnostics control software package was designed to control up to 10 intelligent fast cameras from a single workstation. This central control software is called VIDACS (Video Diagnostics Data Acquisition and Control Software) and the individual camera controller softwares, running in separate PCs, are called EDIDAQ (EDICAM Data Acquisition Software). This package could fulfill all the requirements until OP1.2 and went through numerous improvements and changes. The experience gathered during campaigns required to release a new version of this software package - to ensure high reliability. The previous version of VIDACS graphical user interface can be seen below (Fig 1.)

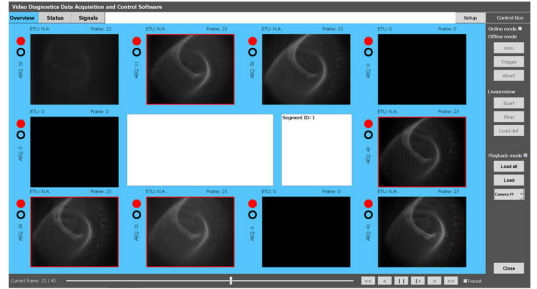

Fig. 1. The main window of VIDACS with the old graphical user interface

\section{New requirements}


In campaign OP1.2 W7-X will provide longer and higher energy plasmas. Video diagnostics has to be ready for this new challenge, especially for storing and archiving more data than before. For this, real-time data streaming is needed to W7-X's Archive also enabling the operators or other researchers to immediately have a look at the results.

Another new feature is the capability to control the trigger-time-event (TTE) unit [4], using a stand-alone GUI. For this purpose the IPP-CODAC team provided the basic API level functions. The idea is to control the TTE unit between shots to be able to change the timing settings: the number of triggers and delays in the available 7 outputs. The TTE control software interface is also capable to read the standard W7-X trigger times, and send them to each individual EDIDAQ via the network for the calculation of the UTC time stamp of the frames for data archiving.

GUI improvements were also required: The old GUI was designed in a way that is was not scalable and less user friendly. Because of the rapid sequence of experiments at $\mathrm{W} 7-\mathrm{X}$, the operators should have the possibility to change settings as fast as possible. Therefore, in the new control software camera groups could be created, and group settings should be applied to any other camera by drag and drop. Another essential need was that users could see the exposure settings both in a graph and in human readable numerical format; the camera sensor readout times should be also shown in the same graph. The users should be able to change the region of interest setting visually with mouse, too.

Another important requirement is to make use of the cameras' event detection capability. The imaging diagnostics groups at W7-X has defined a data structure that all event detection systems can use for sending event info to a so-called event display, where events can be seen from different diagnostics in a common display. [5]

\section{The new system}

The whole software package was fully rewritten using newer version of development environment and compilers. For the development we used Qt framework 5.10.0 with Microsoft Visual C++ 2015 compiler. The bottleneck in choosing the development environment version was the HDF5 library we are using (v1.8.15): is supports up-to the Visual C++ 2015 compiler. Similarly to the old version the new software package is divided into two parts. The central software, which controls all the cameras and has a graphical user interface, is called VIDACS2, and the individual camera control software is EDIDAQ2, running in separate PCs, similarly to the old version.

The main goal was to make VIDACS2 more userfriendly, and to meet this requirement the user interface was redesigned. Now it can run in a 4-screen layout as well; in W7-X control room the video diagnostics has 4screen terminals, and this new layout allows the operator to be able to see all the necessary information simultaneously without switching or opening other application windows. A screenshot can be seen about VIDACS2 below. (Fig 2.)

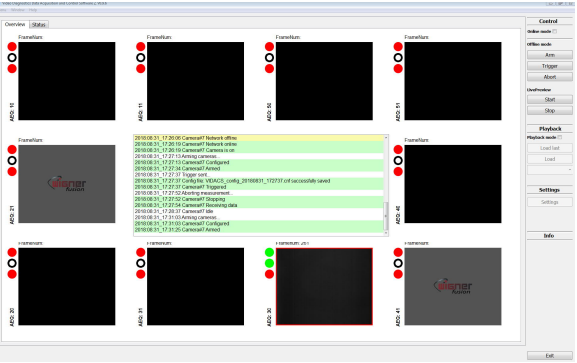

Fig. 2. The main window of VIDACS2 with the new graphical user interface

On the Settings window the user now can see graphically the exposure cycle settings. This makes the camera settings definitely easier. It is also possible to zoom in and out or change the scales of each axis. The users can also save the graph into an image file. An example can be seen in Fig 3. On this settings page the sensor readout times are also displayed, which gives the users a feedback about how much the camera sensor bandwidth is used up by the current settings.

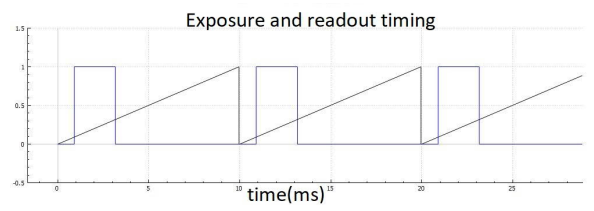

Fig. 3. Exposure (slope) and readout (square) timing on the settings page of VIDACS2

On the ROI settings page, the users now can move the ROIs on the sample image, which makes the positioning of the ROIs significantly easier and faster. These settings can be also saved as an image file. (Fig 4.)

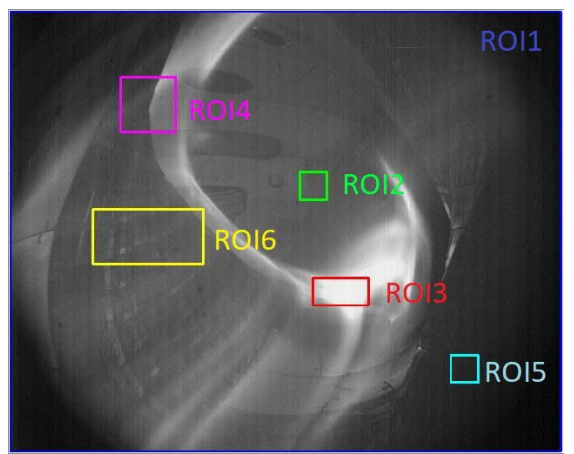

Fig. 4. Region of interest setup on the settings page of VIDACS2 
Most of the settings are now displayed in a more user friendly format, for example sample times or exposure start time can now be defined in millisecond format instead of ETU (Edicam Time Unit, 1 ETU $=100 \mathrm{~ns}$ ). Additionally, there are settings which are checked while the user is typing them, for example ROI sizes are updated automatically if there was a change elsewhere in a related field.

The status window was also re-structured, unnecessary status information was removed, and only camera system temperatures are displayed now. Temperature ranges were also defined and indicated by colors. Status windows show temperatures with green under 50 Celsius, yellow under 55 Celsius and with red above 55 Celsius. If the user double clicks onto the panels it opens a separate window which displays more detailed individual camera status info. This window can be seen below (Fig 5.)

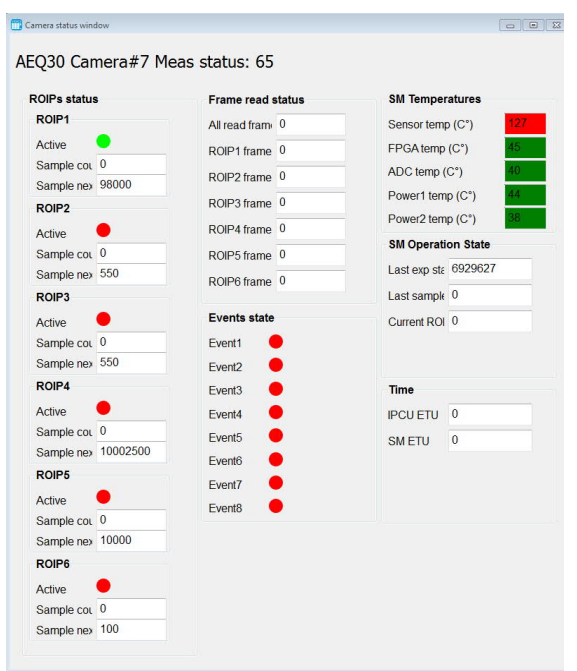

Fig. 5. Status window of VIDACS2 showing all necessary information for one EDICAM

EDIDAQ2 got a new console interface so even if the users cannot interact directly with this software, they can now see exhaustive information about the current camera system status. In this new version there are status indicators that can also change their colors according to the current measurement state which helps to identify the system status by a short glimpse. The software is heavily multi-threaded to ensure the parallel execution of different tasks, for example the down-sampled live image stream to VIDACS2, real-time data streaming to the W7$\mathrm{X}$ Archive and status display. HDF5 files now contain more information about the measurements, they now store all the settings for the current measurement as well, resulting in a single measurement file containing all the data and metadata. This also makes post processing easier.

Another new feature is the real-time data streaming to the W7-X's Archive, also done by the EDIDAQ2s. They receive trigger time stamps from the TTE Unit Controller software, this will be described later. At W7-X there are seven consecutive "standard triggers" in a discharge sequence; the video diagnostics is using three of them t0, $\mathrm{t} 1$ and $\mathrm{t} 6$. The $\mathrm{t} 0$ is the experiment start trigger, used to initialize our system; t1 (exactly $60 \mathrm{~s}$ after t0) is the plasma start trigger, used to start the cameras; and t6 is the experiment end trigger, used to stop our system. Real-time data streaming requires precise time stamps, which are derived using $t 1$. To ensure the reliability of the streaming, the to trigger time is not only read from the TTE unit, but also from the experimental programs web page of W7-X at every measurement startup phase; then, even if the t1 trigger time stamp is missing due to an error, we can substitute it by t0 plus $60 \mathrm{~s}$. This back-up process does not influence the precision of the time stamps because the time between t 0 and $t 1$ is exactly $60 \mathrm{~s}$ with ns accuracy.

\section{Other developments}

To meet the new system's requirements and to make use of the capabilities of our programmable TTE Unit we developed a so called TTE Unit Controller software which is capable to configure the unit on-the-fly, read the trigger times and broadcast them to EDIDAQ2 software as well.

TTE Unit Controller is a GUI application which is also running in the VIDACS2 PC because this central control PC already has a connection to the TTE Unit, and multiple connections are not allowed. Video diagnostics operators can set all the 8 output channels of the TTE device; in our design one channel is dedicated to provide multiple triggers relative to $t 1$. The operators can set the number of triggers (up to five) and the delays for each trigger. It is also possible to generate test triggers on the outputs, so that the video diagnostics system can be tested with hardware triggers at any time without plasma operation. This software also connects automatically to all running EDIDAQ2s via the network using TCP protocol, to be able to send the trigger time stamps as soon as they are available; this is necessary for real-time data streaming into the W7-X Archive. The GUI of the TTE Unit Controller can be seen below (Fig 6.) 


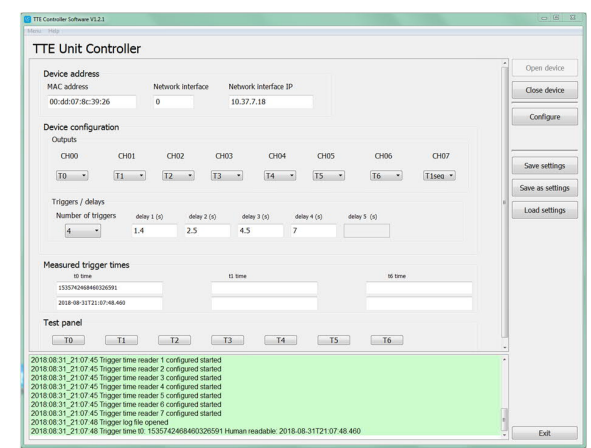

Fig. 6. TTE Unit controller main window

The video diagnostics system has three high-speed fast cameras as well which are controlled separately. To make the control and data saving much more convenient, a server-client based software package was created, similarly to VIDACS2-EDIDAQ2. With this software package the user can set file names and the amount of frames that the user wants to save for each camera, and the client software in each camera PC will save the data in HDF5 file format accordingly. The server software can also ask the client software to start the camera controller software again after the data saving is finished.

\section{Further developments}

Further developments include for example to prepare the system for pulse train measurements. These kind of measurements contains example.g. ten "mini" shots with $30 \mathrm{~s}$ time gap where not all of the standard triggers are generated all the time. Hence, presently our system is only capable to record the first shot in a pulse train because for rearming the system we are not getting the necessary triggers. This can be overcome by further software developments, e.g.by making the system automatically rearmable.

Event distribution among other diagnostics is also an upcoming development which will be tested over the next years. Imaging diagnostic groups at W7-X worked out a specification of the format in which the diagnostics should send their events to a so-called event display (foreseen to be a PC) over the network. The event description should be sent in JSON format, which is a text-based data transmission format for asynchronous network communication. The events are post processed in the event display PC and displayed real-time. This event structure also contains data about which camera detected the event, when it occurred or what was the maximum pixel intensity level.

Presently, the real-time data streaming only contains image raw data. In future software versions a parameter stream will be also sent to the W7-X Archive. This parameter stream can contain all the parameters that are relevant to the images, for example, ROI size, position, settings information or even event states.

This new software package can be also adapted to other fusion experiments which uses EDICAM fast cameras. A similar version of this system is being developed for JT-60SA experiment as well, with modified interfaces to be able to communicate with JT-60SA experiment control systems.

\section{Summary}

The new video diagnostics control software package meets all the new requirements and is continuously evolving during OP1.2 campaign. The main new features are the real-time data streaming to the Archive, GUI improvements to make the software much more user friendly, stable and the capability to detect events and display them in VIDACS2. The system was also capable to run up to 6 ROIs simultaneously, 1 ROI in full resolution typically with $100 \mathrm{~Hz}$ and 5 ROIs with smaller size and much higher frequency, typically $10-20 \mathrm{kHz}$ thus it was sutiable to make measurements that can be correlated with other diagnostics, for example the beam emission spectroscopy diagnostics, while the full frame monitoring was also done simultaneously. Further improvements also include event distribution to a central event display as well. This software package can also adapted to other fusion experiments which uses EDICAM fast cameras, a similar system is being developed for JT60SA experiment as well.

\section{Acknowledgments}

This work has been carried out within the framework of the EUROfusion Consortium and has received funding from the Euratom research and training programme 20142018 under grant agreement No 633053. The views and opinions expressed herein do not necessarily reflect those of the European Commission.

\section{References}

[1] A. Dinklage et al., Nature Phys. 14, 855 (2018)

[2] S. Zoletnik et al, Fus. Eng. Des. 88 (2013) 1405

[3] T. Szabolics et al., Fusion Eng. and Design 123 (217) 995-1000

[4] J. Schacht et al., IEEE-NPSS Real Time Conference (RT) (2016)

[5] Sitjes A. et al., Fusion Science and Technology 74 (2018) 116-124 